\title{
A User-Centered Approach to Design a Mobile Application for Chronic Pain Management
}

\author{
Yingdong Liu ${ }^{1}$, Benoît Encelle², Karim Sehaba ${ }^{3}$ \\ ${ }^{1}$ University of Strasbourg, LISEC, UR 2310, Strasbourg, France \\ ${ }^{2}$ University of Lyon, University Lyon 1, CNRS, LIRIS, UMR5205, Lyon, France \\ ${ }^{3}$ University of Lyon, University Lyon 2, CNRS, LIRIS, UMR5205, Lyon, France
}

Corresponding Author Email: yingdong.liu@etu.unistra.fr; benoit.encelle@liris.cnrs.fr; karim.sehaba@liris.cnrs.fr

https://doi.org/10.18280/mmc c.811-405

Received: 10 August 2020

Accepted: 22 October 2020

\section{Keywords:}

chronic pain/low back pain management, usercentered design, CHI/UX assessment, mobile application

\begin{abstract}
This paper presents a user-centered approach for the design/re-design of a mobile application for chronic pain management, with a focus on chronic low back pain, sometimes referred to as "invisible disability". Within the framework of a multidisciplinary project involving physicians, patients and computer scientists, the work presented here describes firstly an identification and representation process of the needs/expectations of the users of such an application (patients/physicians), based on a data-driven persona development method and, secondly, an evaluation process of the user experience (UX) of the current version of the application, using among others usability tests. The aim of the approach is to establish (re-)design guidelines in order to improve this kind of application.
\end{abstract}

\section{INTRODUCTION}

The work presented in this article was carried out within the framework of the MIA Healthcare project involving the SATT Pulsalys (Pulsalys, SATT (Society for Acceleration of Technology Transfert); Lyon Saint-Etienne), the pain center of the Saint Jean de Dieu hospital in Lyon, the LIRIS (LIRIS, Laboratory of informatics in image and systems of d'information, UMR CNRS 5205, university of Lyon) computer science laboratory and the company santéNet specialized in the development of applications dedicated to pain management. The objective of this project is to develop a mobile application dedicated to the management of chronic pain. This application will assist $1 /$ the doctor in understanding the causes, effects and consequences of pain specific to each patient and $2 /$ the patient in managing his pain through personalized advice. In this context, this article presents a usercentered approach for the design/re-design of such apps, involving both physicians specialized in pain and patients with chronic pain. This approach consists of two steps.

The first step consisted in identifying the needs/expectations, attitudes/behaviors of future users (physicians and patients) of such a kind of application and, in order to implement an efficient and cost-effective usercentered design, to identify typical user profiles. A quantitative (questionnaire) and qualitative (interviews) study, followed by a data analysis/clustering process were conducted, leading to the development of typical user profiles (personas).

The second step of the approach consisted in the evaluation of the user experience (UX) on the current MIA Healthcare application. This UX evaluation was conducted using a questionnaire, a benchmarking of obtained results, and field experiments (usability tests). The aim was, on the one hand, to optimize UX by identifying and correcting design issues and, on the other hand, to establish a taxonomy/classification of these issues by synthesizing and grouping them.
Our initial focus was on the management of Low Back Pain (LBP), although the aim of the application is to take into account all types of chronic pain. This choice was motivated by the magnitude of this disease, which affects more than 540 million people [1]. In France alone, the prevalence of LBP (over a lifetime) in the adult population varies according to studies from $66 \%$ to $75 \%$ [2]: between two thirds and three quarters of people will suffer from LBP at least once in their lifetime. For France, the annual direct medical financial cost is estimated at more than one billion euros, with indirect costs estimated to be 5 to 10 times higher according to $[3,4]$. Indeed, LBP is one of the main causes of activity limitation and absence from work, with heavy economic repercussions on individuals, their families, industry and governments [5]. LBP, sometimes described as an "invisible disability", is now the leading cause of disability worldwide [6].

Section 2 first presents a concise state of the art on existing applications (similar to the one under consideration) and their limitations, and then presents the specificities of the $M I A$ Healthcare project and its objectives. Section 3 details our contribution: the approach used, and the main results obtained. We then discuss this approach and results (section 4), and finally conclude.

\section{RELATED WORKS AND SPECIFICITIES OF THE MIA HEALTHCARE PROJECT, OBJECTIVES}

Applications dedicated to pain management are more and more numerous (Pain Therapy, Pain sense, Mon Coach Douleur, CatchMyPain, etc.). However, the involvement of healthcare professionals in the development of this kind of application is considered insufficient $[7,8]$ and most of these applications lack the ability to adapt and customize their features to end-users. 
As a result, the MIA Healthcare project (see Figure 1) follows a user-centered design approach, involving physicians and patients, for the development of a personalized monitoring application that takes into account the specificities of each patient (i.e. pain, profile). To this end, the MIA Healthcare application plans to question patients on a daily basis about the characteristics/factors related to their chronic pain, such as pain intensity, feeling of fatigue, quality of sleep, psychological state, medication compliance, etc. The application will also be used to monitor and advise patients on the quality of their sleep. The use of such data should make it possible to $1 /$ promulgate to a given patient, using a Chatbot, personalized advice in order to help him/her manage his/her pain and thus improve his/her quality of life and $2 /$ produce a medical pain logbook for health professionals, to better understand a patient's condition and its evolution, leading to better diagnoses and treatments.

Within the framework of a user-centered design, our goal in this project is to provide tools to guide the design/re-design of such an application. In this way, end-user needs have to be identified/prioritized. In order to formulate design/re-design guidelines, we need to a) identify typical profiles and b) evaluate the user experience on the current MIA Healthcare application.

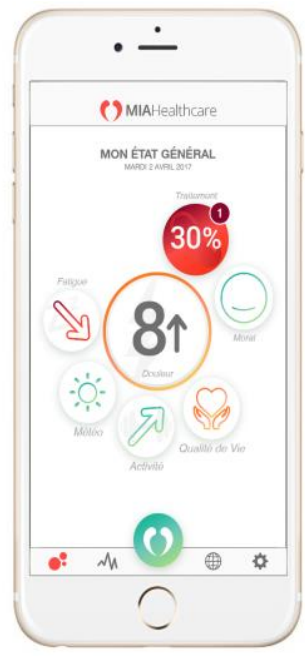

Figure 1. Main screen of the MIA Healthcare application

\section{DESIGN PROCESS AND MAIN RESULTS}

\subsection{Needs/expectations identification and personas development}

\subsubsection{Objectives and expected results}

Our goal is to better understand the future users of a pain management application, by studying potential correlations between the different identified profiles of doctors/patients, their needs/expectations and their interest in such an application. These profiles will include classic sociodemographic data, information on behaviors/attitudes towards new technologies in general and on such type of application, the type/frequency of pain (for patients), etc. The objective here is to study the needs/expectations for a chronic pain management application in general, by not taking into account the existing MIA Healthcare app (so as not to bias the study).
In terms of expected results, functional needs will have to be identified, weighted and ordered, and typical user profiles will have to be identified. The identification of a limited set of typical user profiles allows the design process to be undertaken at a lower cost (in terms of human, temporal and financial resources): this design is in fact centered on the main needs/expectations of the identified profiles. Concretely, the results of such a study should guide the design/re-design of a chronic pain management application (whatever it is).

\subsubsection{Means and implementation}

Quantitative (questionnaires) and qualitative (interviews) studies were conducted on the target population (patients/physicians).

Functional needs/expectations were then specified and personas (typical profiles) were developed on the basis of these studies. The concept of persona was introduced by Cooper [9]. The benefits of using personas are as follows:

- To share a specific understanding and a unique representation of each identified user group/type of users (persona).

- To guide/validate/prioritize suggested solutions and related IT developments, with regard to their adequacy for each persona.

- To provide a human "face" in order to create empathy.

In the following sub-sections, we detail the means used to conduct these studies and their implementation.

Means: Questionnaires. In order to create the patient and doctor questionnaires, whose results integrate the persona development process, we used:

1/ Five types of variables, recommended by Cooper et al. [10] to identify the goals and behaviors of users according to a given product (here the product is the targeted application):

- Activities: what the user does, frequency and volume for, in our case, the management of chronic pain.

- Attitudes: what the user thinks, his/her positioning/feeling/opinion on the product domain (here the e-health and pain management applications)

- Aptitudes: what education and training the user has and his ability to learn.

- Motivations: why the user is engaged in the product domain.

- Skills: user abilities related to the product domain and technology (ability to use new technologies, mobile applications).

For example, for the patient questionnaire, the variable "activities" should make it possible to understand what patients actually do to manage/treat pain and how often. As this variable cannot be measured directly, it can be estimated by asking patients about their sports practice, the type and frequency of medical consultations, etc. This variable can also be used to estimate the frequency of pain management.

2/ classic sociodemographic variables, to understand the overall context of users: gender, age, level of education, field of activity.

The questionnaires created include about 30 questions for the patient and about 20 questions for the doctor. Most of these questions are closed-ended, with some being conditional (asked depending on previous answers). 
Means: Interviews. In addition, in order to better understand the specific context of future users of such application and thus complete the results from the questionnaire analysis, interviews of about ten minutes were conducted with physicians and patients. The questions asked covered topics similar to those addressed in the questionnaires. These interviews will allow us to refine our understanding of, for example, the daily management of chronic pain and compliance with treatments, attitudes towards this type of application, functional expectations/needs, and the ability to use new technologies.

Implementation. For the sample used to conduct these studies, the questionnaires had a total of 99 respondents: 67 patients and 32 physicians. Interviews were conducted with three pain physicians, a psychologist and 12 patients.

Needs/expectations identification

A first list of interesting features has been identified following a state of the art and an analysis of existing similar applications. This list of features was then evaluated and completed by pain specialists, including some features dedicated to the medical profession.

In order to weight and order these features, and in order to have a relatively short and easy to fill out questionnaire, two questions were developed: the first asks for the "top 5" of the most important features in the previously established list (check boxes), and the second invites the participant to complete the proposed list (open question on missing essential features).

\section{Persona development}

A criticism is often made on classical methodology of persona development, indicating that the personas are often not or too few based on real user data [11]. To avoid this pitfall, we propose to use a data-driven method of persona development [12] and to use a clustering method to determine, from the collected data (answers to questionnaires), the optimal number of user groups (i.e. number of personas) for each category of users (patient personas, physician personas) and the representative/discriminatory variables for the groups formed. More precisely, a data clustering method aims at dividing a data set (here the responses of the patient/physician subjects to their respective questionnaire) into different homogeneous groups (clusters), in the sense that the data of each group share common characteristics. Given that the variables measured by the questionnaire are derived from standard variables [10] not directly observed, and that we wish to obtain an optimal number of user groups/classes for each user category (patient/physician), we used the statistical method LCA (Latent Class Analysis) [13-15] to perform the clustering using R software.

\subsubsection{Results}

Questionnaire analysis. The main results of the analysis of the patient questionnaire are presented below. $80.6 \%$ are women, $19.4 \%$ are men. $85.1 \%$ of the participants are between 30 and 60 years old. $53.8 \%$ have at least a "baccalauréat" (i.e. high school diploma). $49.25 \%$ of the participants suffer from multiple pains. $91 \%$ own a smartphone and $77 \%$ report using mobile applications several times a day. Finally, $83.6 \%$ of the respondents say they are interested in the application.

The main results following the analysis of the physician questionnaire are: $53.1 \%$ are men, $46.9 \%$ are women. $90.6 \%$ of physicians have been managing chronic pain for more than 5 years. $65.7 \%$ often use mobile applications. $46.9 \%$ of physicians have already used a smartphone in a professional setting and $59.4 \%$ declare that they would be willing to use a mobile application in a professional setting. $81.3 \%$ said they would be interested in the application.

Regarding the main functional expectations/needs, the results on the patient side are as follows (only the first five features are presented): 1/Proposal of physical exercises/massages $(80.6 \%), 2 /$ Assessment of morale, stress, fatigue, sleep $(73.1 \%), 3 /$ Constitution of a medical pain logbook (68.7\%), 4/Community of patients (specific social network) (59.7\%), 5/Information on chronic pain $(50.7 \%)$.

Physician's side: 1/Assessment of morale, stress, fatigue, sleep $(84.4 \%), 2$ Constitution of a medical pain logbook (68.8\%), 3/Proposal of physical exercises/massages (53.1\%), $4 /$ Information given on the effectiveness of a given treatment (46.9\%), 5/Remote patient monitoring, alarm in case of anxiety and depression risks. It should be noted that only feature 5 / is dedicated to the medical profession.

These results show that some pain specialists are unwilling to use a mobile application in a professional setting. It would therefore be interesting to study why more precisely. Physicians and patients share many of the same needs. Indeed, the three features considered most important are found on both sides, in a different order. Cumulatively, the assessment of morale, stress, fatigue, sleep comes first, followed by the constitution of a medical pain logbook and the proposal of physical exercises/massages. These results underline the importance of evaluating the patient's state (morale, stress, etc.) and its follow-up/evolution (medical pain logbook) for these two types of users. Special attention must therefore be paid to the development of these features.

Persona development. The LCA [13-15] method used on the data from the questionnaires together with the BIC (Bayesian Information Criterion) and AIC (Akaike Information Criterion) information criteria enabled us to determine the optimal number of clusters (called classes below) that corresponds to the personas that have to be created for each categories of users (patient/physician). An analysis of these classes guides the description of these personas. Then, interviews (qualitative data) were used to refine the development of these personas.

\section{Description of the classes obtained}

The results obtained led us to consider two classes for the patient side and only one for the physician side. We detail here only the two patient classes. The first class represents $72.7 \%$ of the participants and the second $27.3 \%$.

With regard to the values of discriminating variables among these classes, the first class tends to have more intense and pervasive pain (intense and extremely intense pain: $55.4 \%$; omnipresence: $100 \%$ ) than the second (intense and extremely intense pain: $0 \%$; omnipresence: $39.77 \%$ ).

In addition, and not surprisingly, participants in the first class consult physicians more often than those in the second (89.73\% consult general practitioners more than four times a year versus $39.77 \%$ for the second; $16.5 \%$ for the first class never see specialists versus $54.53 \%$ for the second).

Concerning the common values of variables, almost all participants have been suffering from pain for more than a year (97.94\% for the first class, $100 \%$ for the second). $68.7 \%$ of the participants do sport at least once a week $(61.66 \%$ for the first, $87.31 \%$ for the second). The participants are more interested in sharing information about their chronic pain $(95.84 \%$ for the first and $72.78 \%$ for the second). As an example, Figure 2 shows the persona card representing the first class. 

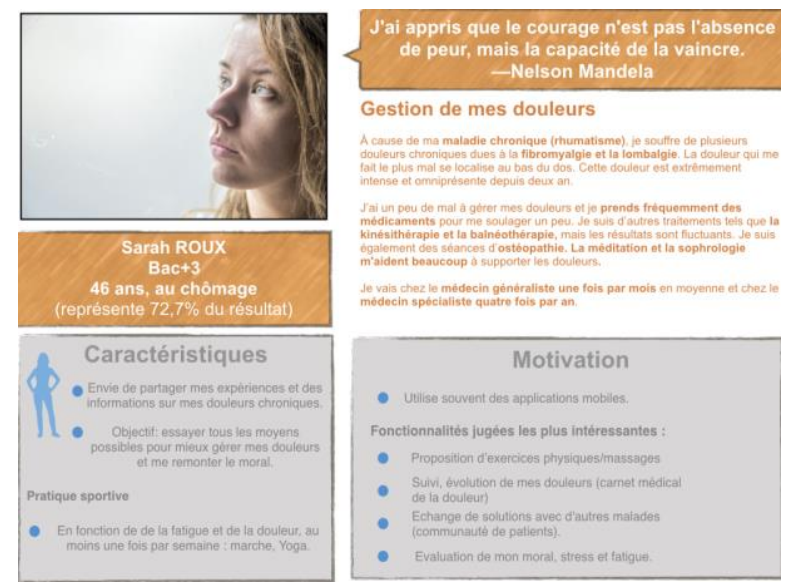

Figure 2. Person for the first class of patients

\subsection{Analysis of the current version of the application: evaluation of the user experience, usability testing}

\subsubsection{Objectives and expected results}

An analysis to assess some user experience factors on the current version of the MIA Healthcare application was conducted. The results obtained will be used to identify the application's strengths/weaknesses and any usability issues encountered. Overall, results will be used to improve the user experience, by improving the ergonomics/user interface of the application.

\subsubsection{Means and implementation}

Means. In order to assess and situate user satisfaction, the UEQ questionnaire was used [16]. This questionnaire evaluates the user experience using six scales:

- Attractiveness: Overall impression of the product. Do users like or dislike it?

- Perspicuity: Is it easy to get familiar with the product and to learn how to use it?

- Efficiency: Can users solve their tasks without unnecessary effort? Does it react fast?

- Dependability: Does the user feel in control of the interaction? Is it secure and predictable?

- Stimulation: Is it exciting and motivating to use the product? Is it fun to use?

- Novelty: Is the design of the product creative? Does it catch the interest of users?

To situate the evaluation of the user experience, a benchmarking tool containing the results of UEQ questionnaires for 246 different products was used [17]. This tool classifies the product evaluated on each scale using 5 categories: excellent (in the $10 \%$ best results), good (10\% are better, $75 \%$ worse), above average (25\% are better, $50 \%$ worse), below average (50\% are better, $25 \%$ worse), bad (in the $25 \%$ worst results).

To assess usability, usability tests were conducted [18, 19]. For this purpose, typical use case scenarios of the application have been established, based on the main features previously identified (see previous section). For instance, a scenario for the user category "patient", focusing on the feature "Constitution of a medical pain logbook", is the following: "You are experiencing severe pain in your lower back and legs. You assume that this pain is strongly weather-related (wet weather). Record this information on the application".

Implementation. 6 scenarios for usability testing were identified. An evaluation protocol was set up, inspired by conventional usability testing [18, 19], with a fourth step aimed at evaluating the user experience (via the UEQ questionnaire). Protocol steps are the following:

1. Collecting of socio-demographic data: age, new technology familiarity, professional activity, etc.

2. Getting started with the application: a given short length of time to discover the application. During his/her app discovery, the subject is invited to think aloud (verbalizing thoughts as moving through the user interface)

3. Carrying out usability tests (scenarios) with the "think aloud" method.

4. Filling out the UEQ questionnaire.

5. Debriefing. Questions on the perceived interest of the proposed application.

Due to a lack of time and some difficulties in accessing both categories of users, the evaluation was only conducted with patient-type users (access to pain specialists being more difficult). 10 people suffering from chronic low back pain and owning a smartphone took part in the evaluation. The participants were aged between 29 and 70 years $(\mathrm{M}=48.8$ years).

\subsubsection{Results}

Analysis of the usability test results revealed 20 usability issues, which have been classified using the heuristic evaluation criteria proposed by Nielsen [18]. We give below these different criteria, along with the number of issues for each criterion (in brackets) and with an example of an issue associated with each criterion: issues with visibility of system status (5) (e.g. the operations remaining to perform a task are not indicated (e.g. to inform about a pain, the user can easily forget to inform about its location after indicating its intensity)); issues with the match between system and the real world (4) (e.g. the Chatbot's language level may not be adapted to the user, its behavior may be strange (e.g. not saying 'hello' at first)), issues with user control and freedom (3) (e.g. actions cannot be modified or canceled), issues with consistency and standards (4) (e.g. the text size is too small); error prevention issues (1) (lack of user instructions to achieve his or her goal); recognition issues (3) (e.g. the system does not refer to the user's goal).

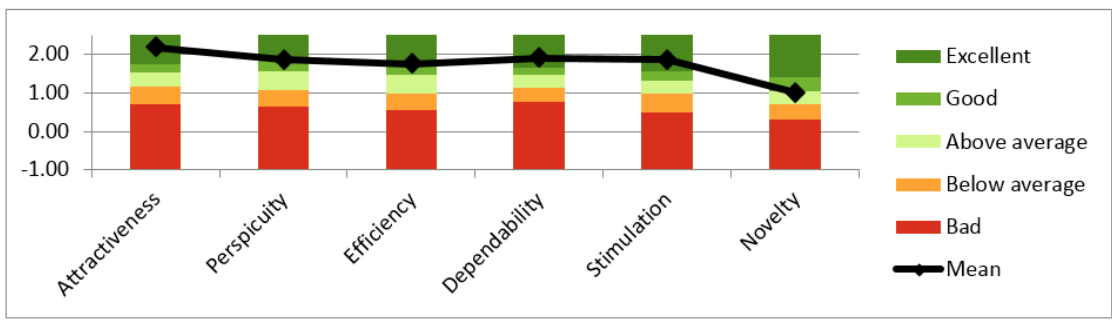

Figure 3. Benchmarking of the application 
Next, re-design proposals and guidelines based on the preceding issues were suggested. These results led to user interface improvements, to minimize user's cognitive load.

Figure 3 presents the results of the UX evaluation (fourth step of the protocol).

The evaluations of the " Attractiveness", "Dependability" and "Stimulation" qualities are "excellent", the "Perspicuity", "Efficiency" and "Novelty" qualities are "good". It appears that the "Novelty" quality score is the lowest: the application may suffer from a lack of originality.

\section{DISCUSSIONS}

The results obtained and presented are drawn from analyses based on patient/physician samples which may be not representative of targeted populations. This is mainly due to the difficulties of accessing the targeted populations (patients with chronic pain - chronic low back pain, specialist doctors). As a consequence, these results should not be generalized, but should be considered in the context of the above studies.

More specifically, although the respondents to the needs identification questionnaire were relatively numerous and came from different places (members of chronic pain associations, chronic pain sufferers who had registered on the SantéNet website, etc.), interviews (patients/physicians); usability tests; UEQ questionnaires were conducted with doctors/patients from the pain center of the Saint Jean de Dieu hospital. This single source may possibly introduce sampling bias.

Moreover, more specialized questionnaires could have been under consideration for the UX evaluation. For instance, the MARS questionnaire [20] is designed to assess the quality of healthcare mobile applications. However, we have chosen to use a fairly general and widely used questionnaire for evaluating the UX, as the use of a (too) specialized questionnaire can lead to a lack of reliability. To continue with the MARS questionnaire, it was clearly designed for the evaluation of mobile applications related to mental health. Therefore, the applicability and reliability of MARS in other health domains remains to be demonstrated [20].

\section{Conclusion and perspectives}

This article presents a user-centered approach to the design of a chronic pain management mobile application for both chronic pain sufferers and physicians. This approach was developed as part of a project involving IT researchers, designers/developers of mobile health applications and practitioners (pain specialists). The objective of this approach is to establish design/re-design guidelines for this kind of application.

A first contribution consists of an identification and representation process of needs/expectations, attitudes/behaviors of typical users of a chronic pain management application. In order not to introduce bias, this process voluntary doesn't take into account the MIA Healthcare mobile application. We first detail the identification phase and then describe the phase leading to the development of typical user profiles (personas).

The second contribution consists of an evaluation and benchmarking process of the user experience (UX) on the current version of the application. This process is detailed (UX questionnaire, usability test development, evaluation protocol, result analysis). If the results presented are specific to the $M I A$
Healthcare application, the suggested approach for UX evaluation: questionnaire/benchmarking tool, construction/analysis of usability tests, evaluation protocol used, is generic.

The main results are as follows:

- The main needs/expectations of the two categories of targeted users (patients/physicians) regarding a chronic pain management application are similar (assessment of morale, stress, fatigue, sleep; constitution of a medical pain logbook; proposal of physical exercises/massages).

- The segmentation of users (typical profiles) by category is as follows: 2 patient classes (differences are on pain characterization/frequency, type/frequency of doctors consulted), 1 physician class.

Future work includes firstly studies on broader samples, representative ones, in order to consolidate or not the findings. It would also be interesting to evaluate the UX of the current application for the health professional's category.

Secondly, it would be also interesting to study the real uses of the application, to complement the suggested redesign approach. This kind of study could be conducted in a "nonintrusive" way (subject to user's agreement), by collecting and analyzing interaction traces. These interaction traces represent the history of interactions between users and the application (feature usages, data inputted, etc.) and provide insights on real users/uses (most used/less used features, trouble encountered, etc.).

\section{REFERENCES}

[1] Hartvigsen, J., Hancock, M.J., Kongsted, A., Louw, Q., Ferreira, M.L., Genevay, S. (2018). What low back pain is and why we need to pay attention. The Lancet, 391(10137): 2356-2367. https://doi.org/10.1016/S01406736(18)30480-X

[2] Quelques chiffres sur la lombalgie et le mal de dos, Lombalgie. Disponible sur: http://www.lombalgie.fr/comprendre/quelques-chiffres.

[3] Fassier, J.B. (2011). Prévalence, coûts et enjeux sociétaux de la lombalgie. Revue du Rhumatisme, 78: S38-S41. https://doi.org/10.1016/S11698330(11)70007-2

[4] Maladie, A. (2017). Risques professionnels: une collection pour mieux comprendre, Risques professionnels : une collection pour mieux comprendre rapport 2017 : la lombalgie liée au travail. Disponible sur: https://assurance-maladie.ameli.fr/qui-sommesnous/publications-reference/assurance-maladie-risquesprofessionnels/rapports-sante-travail.

[5] Duthey, B.B. (2013). Background paper 6.24 low back pain. Priority medicines for Europe and the world. Global Burden of Disease (2010),(March), pp. 1-29.

[6] Vos, T., Allen, C., Arora, M., Barber, R.M., Bhutta, Z.A., Brown, A. (2016). Global, regional, and national incidence, prevalence, and years lived with disability for 310 diseases and injuries, 1990-2015: a systematic analysis for the Global Burden of Disease Study 2015. The lancet, 388(10053): 1545-1602. https://doi.org/10.1016/S0140-6736(16)31678-6

[7] Alexander, J.C., Joshi, G.P. (2016). Smartphone applications for chronic pain management: a critical 
appraisal. Journal of Pain Research, 9: 731-734. https://doi.org/10.2147/JPR.S119966

[8] Rosser, B.A., Eccleston, C. (2011). Smartphone applications for pain management. Journal of Telemedicine and Telecare, 17(6): 308-312. https://doi.org/10.1258/jtt.2011.101102

[9] Cooper, A. (1999). The Inmates Are Running the Asylum. USA: Macmillan Publishing Co., Inc.

[10] Cooper, A., Reimann, R., Cronin, D., Noessel, C. (2014). About Face: The Essentials of Interaction Design. John Wiley \& Sons.

[11] Pruitt, J., Grudin, J. (2003). Personas: practice and theory. In Proceedings of the 2003 conference on Designing for User Experiences, pp. 1-15. https://doi.org/10.1145/997078.997089

[12] McGinn, J., Kotamraju, N. (2008). Data-driven persona development. In Proceedings of the SIGCHI Conference on Human Factors in Computing Systems, pp. 15211524. https://doi.org/10.1145/1357054.1357292

[13] Collins, L.M., Lanza, S.T. (2009). Latent class and latent transition analysis: With applications in the social, behavioral, and health sciences.

[14] Nylund, K.L., Asparouhov, T., Muthén, B.O. (2007). Deciding on the number of classes in latent class analysis and growth mixture modeling: A Monte Carlo simulation study. Structural Equation Modeling: A
Multidisciplinary Journal, 14(4): 535-569. https://doi.org/10.1080/10705510701575396

[15] Nylund-Gibson, K., Choi, A.Y. (2018). Ten frequently asked questions about latent class analysis. Translational Issues in Psychological Science, 4(4): 440-461. https://doi.org/10.1037/tps0000176

[16] Laugwitz, B., Held, T., Schrepp, M. (2008). Construction and evaluation of a user experience questionnaire. In Symposium of the Austrian HCI and Usability Engineering Group, p3. 63-76. https://doi.org/10.1007/978-3-540-89350-9_6

[17] Schrepp, M., Hinderks, A., Thomaschewski, J. (2017). Construction of a benchmark for the user experience questionnaire (UEQ). IJIMAI, 4(4): 40-44. https://doi.org/10.9781/ijimai.2017.445

[18] Nielsen, J. (1994). Usability Engineering. Morgan Kaufmann.

[19] Rogers, Y., Sharp, H., Preece, J. (2011). Interaction Design: Beyond Human-Computer Interaction. John Wiley \& Sons.

[20] Stoyanov, S.R., Hides, L., Kavanagh, D.J., Zelenko, O., Tjondronegoro, D., Mani, M. (2015). Mobile app rating scale: a new tool for assessing the quality of health mobile apps. JMIR mHealth and uHealth, 3(1): e27. https://doi.org/10.2196/mhealth.3422 